\title{
BIBLIOGRAFÍA ESPECIALIZADA DE TRADUCCIÓN \\ SOBRE INTERPRETACIÓN: EL PROYECTO HERMĒNEUS DE \\ PUBLICACIONES DE TRADUCCIÓN E INTERPRETACIÓN DE LA \\ FACULTAD DE TRADUCCIÓN E INTERPRETACIÓN DE SORIA - \\ UNIVERSIDAD DE VALLADOLID (1999-2011) \\ Comité de Redacción del Proyecto Hermèneus ${ }^{1}$ \\ Universidad de Valladolid, España \\ www.uva.es/hermeneus
}

\begin{abstract}
From 1999 up to the present, the Faculty of Translation of Interpreting at the University of Valladolid, Spain, has published three closely related series on Translation and Interpreting, the so-called Proyecto Hermēneus (Hermēneus Project): Hermeneus Journal, the Vertere Monographs, and Disbabelia, a collection of unknown translations. This paper seeks to make some of their features known as well as offer their full bibliographical data.
\end{abstract}

KEY WORDS: Translation and Interpreting, periodicals, Hermēneus Project, Hermèneus, Vertere, Disbabelia, bibliography

\section{RESUMEN}

La Facultad de Traducción e Interpretación de Soria, perteneciente a la Universidad de Valladolid, publica desde el año 1999 hasta la fecha tres series relacionadas entre sí dedicadas a la traducción y la interpretación, el llamado Proyecto Hermēneus compuesto por la revista Hermènens, los monográficos Vertere y las traducciones ignotas Disbabelia. El presente trabajo comentará algunos rasgos de dichas colecciones, y recogerá los datos bibliográficos completos de las mismas.

1 Cristina Adrada Rafael (Universidad de Valladolid); Sabine Albrecht (Friedrich-Schiller Universität Jena - Jena - Alemania); Vivina Almeida Carreira (Instituto Politécnico de Coimbra - Portugal); Susana Álvarez Álvarez (Universidad de Valladolid); Rocío Anguiano Pérez (Universidad de Valladolid); Verónica Arnáiz Uzquiza (Universidad de Valladolid); Larry Belcher (Universidad de Valladolid); Carmen Cuéllar Lázaro (Universidad de Valladolid); Rosario de Felipe Boto (Universidad de Valladolid); Marie Hélène García (Université d'Artois Arras - Francia); Elena di Giovanni (Università di Macerata - Macerata - Italia); Juan Miguel Zarandona Fernández (Universidad de Valladolid). 
PALABRAS CLAVE: Traducción e Interpretación, publicaciones periódicas, Proyecto Hermēneus, Hermèneus, Vertere, Disbabelia, bibliografía

\section{INTRODUCCIÓN}

La revista Entreculturas, gestada en la Facultad de Traducción e Interpretación de la Universidad de Málaga, así como los responsables de su gestión, nos han brindado la amable oportunidad de dar a conocer en sus páginas las publicaciones en torno a la traducción y la interpretación de la Universidad de Valladolid, concediéndonos al mismo tiempo el privilegio de difundir datos y realidades sobre el Proyecto Hermēneus de la Facultad de Traducción e Interpretación de Soria, empresa editora que representamos en calidad de Comité de Redacción. Por estos motivos, nos hemos puesto a la tarea con ilusión y agradecimiento. El resultado de nuestro esfuerzo queda recogido en las tres secciones que se presentan a continuación, cada una de ellas dedicada, respectivamente, a una de las tres colecciones de publicación periódica que constituyen el mencionado Proyecto Hermēneus: la revista Hermèneus, los monográficos Vertere y la colección de traducciones ignotas Disbabelia.

\section{HERMĖNEUS. REVISTA DE TRADUCCIÓN E INTERPRETACIÓN}

Cuando se publicó por primera vez, en la entonces recién inaugurada Facultad de Traducción e Interpretación de Soria (1995), Hermèneus se presentó ante la comunidad científica como una publicación de periodicidad anual de la Universidad de Valladolid de carácter científico, encaminada a la edición de artículos originales, reseñas de libros y otros textos complementarios, todos ellos dentro de los campos de actividad e investigación de la traducción, la interpretación y otras áreas lingüísticas, documentales, literarias y humanísticas afines. La nueva revista nació con vocación multilingüe, por lo que proclamó, igualmente desde el principio, que las lenguas principales de trabajo a las que deberían atenerse los interesados en publicar en Hermèneus serían español, francés, inglés, alemán e italiano. Pero no solo éstas, cualquier otra lengua sería tenida en cuenta también como adecuada para la revista, con la única limitación de encontrar personas con la competencia lingǘstica y conocimientos en la materia adecuados para valorar un artículo en una lengua determinada. Otro fundamento que se adoptó desde el origen de la revista fue la de actuar gobernados por las decisiones razonadas de expertos en las materias 
concernidas, por lo que se buscó contar con un amplio, prestigioso, multilingüe y variado, en cuanto a enfoques, equipo de expertos que constituyeron la columna vertebral de toda su organización: el Comité Científico. En otras palabras, las tres publicaciones del Proyecto Hermèneus cuentan con el privilegio de la colaboración de un amplísimo elenco de especialistas de primera línea, nacionales e internacionales, del área de Traducción e Interpretación y de otras afines.

Muchas cosas han acontecido desde aquel 1999; sin embargo, todo lo fundamental se ha mantenido firme. Siempre se ha acudido puntualmente a la cita, lo que ha provocado el feliz resultado, para autores, investigadores e interesados en general, de cientos de introducciones, artículos, reseñas, traducciones y otros textos de naturaleza variada. Fruto maduro de este esfuerzo, Hermèneus disfruta del reconocimiento que le otorga su pertenencia a las siguientes bases de datos y fuentes documentales, la llamada visibilidad:

1. ISOC del Consejo Superior de Investigaciones Científicas (CSIC) (España). http:/ / bddoc.csic.es:8080/isoc.htmlijsessionid=E093 A9F8

2843F455365BAC8A70D26BA7.

2. LATINDEX. Directorio de Publicaciones Cientificas Seriadas de España, Iberoamérica y Latinoamérica. Dirección digital en: bttp:/ / www.latindex.unam.mx/.

3. MLA International Bibliography / Directory of Periodicals (Estados Unidos). bttp://www.mla.org/bibliography.

4. Linguistics and Language Behavior Abstracts database de Cambridge Scientific Abstracts (CSA) Current Serials Source List (as priority). ProQuest (Estados Unidos). http://wmw.csa.com/.

5. Bibliographie Linguistique / Linguistic Bibliography publicada por Brill Academic Publishers - Koninklijke Brill NV / Comité Internacional Permanente de Lingüística (CIPL). Países Bajos. bttp:// www.ilx.nl/blonline/, www.linguisticbibliography.com.

6. FRANCIS del Institut de L'Information Scientifique (INIST) del Centre Nacional de la Recherche Scientifique (CNRS) (Francia). http://international.inist.fr/ rubrique19.html.

7. Asimismo, los resúmenes (abstracts) se publican en la revista especializada Translation Studies Abstracts de la editorial St. Jerome (Manchester, Reino Unido), y en la página digital relacionada Translation Studies Abstracts. Bibliography of Translation Studies: www.stjerome. co.uk/tsaonline.

8. Igualmente, la base de datos BITRA (Bibliografía de Interpretación y Traducción) de la Universidad de Alicante / Alacant (España): 
ENTRECULTURAS Número 4. ISSN: 1989-5097. Fecha de publicación: 29-01-2012

http:// wmw.ua.es/dfing/tra int/bitra.htm, incluye las publicaciones del Proyecto Hermēneus.

9. British Humanities Index (BHI). ProQuest. Reino Unido. Consulta: bttp:// www.csa.com/factsheets/bbi-set-c.php.

10. Linguistics Abstracts (LABS), de la Universidad de Alberta, Canadá. http:/ / wmw. blackwellpublishing.com/journal.asp? ref $=0267-5498$.

11. Dialnet. Portal de difusión de la producción cientifica hispana. Universidad de La Rioja. bttp://dialnet.unirioja.es

12. European Reference Index for the Humanities (ERIH), de la European Science Foundation (EFS), en la sección "Linguistics". bttp:// www.esf.org/research-areas/bumanities/research-infrastructu res-includingerib/erih-initial-lists.btml.

13. A Bibliography of Literary Theory, Criticism, and Pbilology. Universidad de Zaragoza. filologia inglesa/garciala/ bibliography. btml. bttp:// www.unizar.es/departamentos/

14. Repositorio Español de Ciencia y Tecnología (RECYT) de la Fundación Española para la Ciencia y la Tecnología (FECYT) http:/ / recyt.fecyt.es.

(Esta relación no está cerrada, ya que en el momento actual se está negociando la inclusión de Hermèneus en otras tres bases de datos)

Este listado de recursos y páginas digitales debe conducir, en nuestra opinión, a resaltar dos hechos. En primer lugar, aunque Hermèneus es una revista que se publica en papel, siguiendo la línea tradicional en este aspecto, ello no impide que se difunda en Internet de manera muy efectiva, a texto completo y por partida triple, lo que garantiza de manera completa su difusión y accesibilidad a todos los posibles interesados en conocer sus propuestas: la propia página del Proyecto Hermēneus (www.uva.es/ hermeneus), la página de Dialnet, anteriormente citada, y el Repositorio Español de Ciencia y Tecnología (RECYT), al que también hemos alusión en nuestro listado.

En segundo lugar, y respecto a este último recurso, queremos señalar, en nuestro favor, lo siguiente. Cuando Hermèneus estaba a punto de cumplir su primera década de existencia, la Fundación Española para la Ciencia y la Tecnología (FECYT), entidad pública del Estado Español, nos anunció por carta el 30 de mayo de 2008 que nuestra revista había sido seleccionada para su inclusión, alojamiento y publicación completa en la plataforma RECYT (Repositorio Español de Ciencia y Tecnología), después de un complejo y prolongado proceso de estudio y evaluación. Solamente treinta revistas españolas fueron elegidas de manera definitiva. Y tan solo trece universidades de nuestro país pudieron incluir una publicación propia en dicha selección. Hermèneus, además, 
fue la única revista del área de conocimiento de Traducción e Interpretación que logró superar todos los requisitos de inclusión.

\subsection{REVISTAS PUBLICADAS}

1999 Hermenēus. Revista de Traducción e Interpretación. No 1 (Soria: Facultad de Traducción e Interpretación - Universidad de Valladolid). ISSN: 1139-7489.

2000 Hermenēus. Revista de Traducción e Interpretación. No 2 (Soria: Facultad de Traducción e Interpretación - Universidad de Valladolid). ISSN: 1139-7489.

2001 Hermenèus. Revista de Traducción e Interpretación. No 3 (Soria: Facultad de Traducción e Interpretación - Universidad de Valladolid). ISSN: 1139-7489.

2002 Hermenēus. Revista de Traducción e Interpretación. No 4 (Soria: Facultad de Traducción e Interpretación - Universidad de Valladolid). ISSN: 1139-7489.

2003 Hermenèus. Revista de Traducción e Interpretación. No 5 (Soria: Facultad de Traducción e Interpretación - Universidad de Valladolid). ISSN: 11397489).

2004 Hermenēus. Revista de Traducción e Interpretación. No 6 (Soria: Facultad de Traducción e Interpretación - Universidad de Valladolid). ISSN: 11397489).

2005 Hermenèus. Revista de Traducción e Interpretación. No 7 (Soria: Facultad de Traducción e Interpretación - Universidad de Valladolid). ISSN: 11397489).

2006 Hermenēus. Revista de Traducción e Interpretación. No 8 (Soria: Facultad de Traducción e Interpretación - Universidad de Valladolid). ISSN: 11397489).

2007 Hermenèus. Revista de Traducción e Interpretación. No 9 (Soria: Facultad de Traducción e Interpretación - Universidad de Valladolid). ISSN: 11397489).

2008 Hermenèus. Revista de Traducción e Interpretación. No 10 (Soria: Facultad de Traducción e Interpretación - Universidad de Valladolid). ISSN: 1139-7489). 
2009 Hermenèus. Revista de Traducción e Interpretación. No 11 (Soria: Facultad de Traducción e Interpretación - Universidad de Valladolid). ISSN: 1139-7489.

2010 Hermenèus. Revista de Traducción e Interpretación. N ${ }^{\circ} 12$ (Soria: Facultad de Traducción e Interpretación - Universidad de Valladolid). ISSN: 1139-7489. Director.

2011 Hermenèus. Revista de Traducción e Interpretación. No 13 (Soria: Facultad de Traducción e Interpretación - Universidad de Valladolid). ISSN: 1139-7489.

\section{VERTERE. MONOGRÁFICOS DE LA REVISTA HERMENNEUS}

También desde el mismo principio, Hermèneus se planteó la necesidad, como actividad complementaria -pero ineludible- a su labor de edición periódica de artículos, reseñas y traducciones breves, la publicación de un volumen anejo, de carácter anual, bajo la denominación genérica de Vertere. Monográficos de la Revista Hermèneus. El primer volumen vio la luz igualmente en el mismo año inaugural de 1999, y Vertere, de la misma manera que lo ha hecho siempre Hermenens, ha acudido puntual a su cita anual en forma de un nuevo volumen. La colección no puede calificarse con otros adjetivos que los más propios de la misma disciplina de Traducción e Interpretación: variada e interdisciplinar. Es decir, los volúmenes de Vertere han atendido todos los enfoques lingüísticos, literarios, culturales o históricos, entre otros, propios del área de Traducción e Interpretación; o han albergado la labor tanto de investigadores nacionales como extranjeros (Estados Unidos, Bélgica, Canadá, Portugal).

Todo ello indica con claridad que las publicaciones de este proyecto, y en especial Vertere, han sido en todo momento un foro libre de discusión, donde no se ha discriminado a nadie por sus ideas y donde solo se accede a la publicación siguiendo criterios científicos. La gran variedad de enfoques y temáticas presentes, antes mencionadas, así lo atestiguan.

\subsection{MONOGRÁFICOS PUBLICADOS}

1999 Vertere. Monográficos de la Revista Hermēneus. No 1 (Soria: Facultad de Traducción e Interpretación - Universidad de Valladolid). Título y autor del 
volumen: La traducción de la variación lingüística, Roberto Mayoral Asensio. Prólogo de Eugene Nida, pp. 13-14. ISBN: 84-95099-05-5.

2000 Vertere. Monográficos de la Revista Hermèneus. No 2 (Soria: Facultad de Traducción e Interpretación - Universidad de Valladolid). Título y autor del volumen: Publicidad y traducción, Antonio Bueno García. Prólogo de Raymond van den Broeck, pp. 15-16. ISBN: 84-95099-29-2.

2001 Vertere. Monográficos de la Revista Hermèneus. No 3 (Soria: Facultad de Traducción e Interpretación - Universidad de Valladolid). Título y autor del volumen: Teoría de la traducción, Mariano García Landa. Prólogo de Jean Delisle, pp. 13-16. ISBN: 84-95099-36-5.

2002 Vertere. Monográficos de la Revista Hermèneus. No 4 (Soria: Facultad de Traducción e Interpretación - Universidad de Valladolid). Título y autores del volumen: José María Suárez: Disertación sobre las medallas y monedas antiguas (De Nvmismatis et Nvmmis Antiqvis Dissertatio), Liborio Hernández Guerra y Beatriz Antón Martínez. Presentación de Juan Miguel Zarandona, pp. 11-12. ISBN: 8495099-48-9.

2003 Vertere. Monográficos de la Revista Hermèneus. No 5 (Soria: Facultad de Traducción e Interpretación - Universidad de Valladolid). Título y autor del volumen: Los versos de la muerte de Hélinand de Froidmont. La traducción de textos medievales franceses al español. Miguel Ibáñez Rodríguez. Prólogo de Juan Miguel Zarandona, pp. 11-12. ISBN: 84-95099-70-5.

2004 Vertere. Monográficos de la revista Hermēneus. No 6 (Soria: Facultad de Traducción e Interpretación - Universidad de Valladolid). Título y autora del volumen: Historia de la traducción en la Administración y en las relaciones internacionales en España. Ingrid Cáceres Würsig. Prólogo de Miguel Ángel Vega Cernuda, pp. 17-19. ISBN: 84-95099-71-3.

2005 Vertere. Monográficos de la revista Hermèneus. No 7 (Soria: Facultad de Traducción e Interpretación - Universidad de Valladolid). Título y autor del volumen: Apuntes de bistoria de la traducción portuguesa. Carlos Castilho Pais. Prólogo de Julio César Santoyo, pp. 13-15. ISBN: 84-95099-89-6.

2006 Vertere. Monográficos de la revista Hermèneus. No 8 (Soria: Facultad de Traducción e Interpretación - Universidad de Valladolid). Título y autor del volumen: ¿Cómo traducir clíticos? Modelo general y estrategias específicas a partir del caso 
de la traducción española de los clíticos franceses EN e Y. Kris Buyse. Prólogo: de Mona Baker, pp. 11-12. ISBN: 84-96695-00-X.

2007 Vertere. Monográficos de la revista Hermèneus. No 9 (Soria: Facultad de Traducción e Interpretación - Universidad de Valladolid). Título y autor del volumen: Traducción y Humanismo: panorama de un desarrollo cultural. Roxana Recio (ed). Prólogo: de Juan Miguel Zarandona, p. 11. ISBN: 84-96695-18-2.

2008 Vertere. Monográficos de la revista Hermèneus. No 10 (Soria: Facultad de Traducción e Interpretación - Universidad de Valladolid). Título y autor del volumen: La traducción en periódicos y revistas españolas desde 1900 basta 1965. Antonio Raúl de Toro Santos y Pablo Gancedo. Prólogo: Román Álvarez, pp. 15-23. ISBN: 84x-96695-24-7.

2009 Vertere. Monográficos de la revista Hermèneus. No 11 (Soria: Facultad de Traducción e Interpretación - Universidad de Valladolid). Título y autor del volumen: El léxico traducido: la lexicografía hispano-filipina de los siglos XVI, XVII y XVIII. Joaquín García-Medall. Prólogo: Policarpo Hernández, pp. 15-17. ISBN: 84-96695-31-X.

2010 Vertere. Monográficos de la revista Hermèneus. No 12 (Soria: Facultad de Traducción e Interpretación - Universidad de Valladolid). Título y autor del volumen: Dictionaries and Vocabularies in Spanish and English from 1554 to 1740: Their Structure and Development. Heberto H. Fernández U. Prólogo / Preface: Juan Miguel Zarandona, pp. 17-18. ISBN: 84-96695-45-X.

2011 Vertere. Monográficos de la revista Hermèneus. No 13 (Soria: Facultad de Traducción e Interpretación - Universidad de Valladolid). Título y autores del volumen: Poesía inglesa femenina del siglo XVIII. Estudio y traducción (antología bilingüe). Vicente López Folgado, Ángeles García Calderón, Miguel Ángel García Peinado y Juan de Dios Torralbo Caballero. Prólogo: Antonio Lastra, pp. 11-13. ISBN: 84-96695-53-0.

\section{DISBABELIA. COLECCIÓN HERMËNEUS DE TRADUCCIONES IGNOTAS}

Hermèneus, como su segunda y también ineludible actividad complementaria a su labor de edición periódica de artículos, reseñas, traducciones breves y estudios monográficos, ideó de inmediato una tercera colección de traducciones, bajo la denominación genérica de Disbabelia. Colección 
Herméneus de Traducciones Ignotas. Se trataba de descender de la teoría, de la especulación y del debate de ideas, a la práctica, es decir, a traducir y a invitar a traducir.

En principio, las traducciones de esta serie tendrían un carácter literario en cualquier género en el que las obras originales estén escritas. Tratados u obras de otros temas de carácter humanístico o cultural podrían también ser tenidos en cuenta para su publicación.

Las lenguas de partida podrían ser todas las lenguas del mundo, del presente o del pasado. La lengua prioritaria de llegada sería el español. Las otras lenguas de enseñanza de la Facultad de Traducción e Interpretación de Soria, esto es, francés, inglés, alemán e italiano, podrían también ser lenguas de llegada, si se considerara oportuno.

Por ignotas debía entenderse que este proyecto se plantea ante todo la traducción desde lenguas minoritarias, exóticas, muertas o artificiales que resulten desconocidas, o muy poco conocidas, y que no hayan sido traducidas, o lo hayan sido en muy escasa medida, al español. También se buscaría la traducción de autores que no conozcan tampoco traducción a dicha lengua, o apenas la conozcan, aunque sus libros estén escritos en una lengua mayoritaria o de cultura dominante.

El propósito confeso de esta colección era complementar o suplir un amplio terreno de autores, obras y lenguas de gran interés cultural y lingüístico, pero no comercial para una editorial con exigencias de mercado puramente empresariales. Correr un cierto riesgo, llegar a donde otros no pueden, tal vez, hacerlo, sin olvidarnos de la elevada misión de la traducción, y poner en contacto y dar a conocer culturas y grupos humanos muy separados entre sí por la división de las lenguas. Para nosotros, cuanto más alejados o desconocidos fueran éstos, mayor sería su interés.

Disbabelia apelaría al mito bíblico de la torre de Babel, tan asociado al surgimiento práctico de la necesidad de la traducción y la interpretación, pero en un sentido contrario. No creíamos que la división de las lenguas fuera una maldición, sino un patrimonio irrenunciable de la humanidad que debía ser cuidado con esmero.

Todos estos objetivos, o más bien, ideales, se han cumplido en grandísima medida. El número y atractivo de los volúmenes publicados hasta la fecha así lo evidencia.

\subsection{VOLÚMENES PUBLICADOS}

2000 Disbabelia. Colección Hermèneus de traducciones ignotas. $\mathrm{N}^{\mathrm{o}} 1$ (Valladolid: Servicio de Publicaciones e Intercambio Editorial - Universidad de 
Valladolid). Título y autor del volumen: Daurel y Betón, Anónimo del siglo XIII. Traducción, introducción y notas de Jesús D. Rodríguez Velasco. Lengua de partida: occitano medieval. Prólogo de Juan Zarandona, pp. 13-15. ISBN: 84-8448-011-9.

2000 Disbabelia. Colección Hermèneus de traducciones ignotas. $\mathrm{N}^{\circ} 2$ (Valladolid: Servicio de Publicaciones e Intercambio Editorial - Universidad de Valladolid). Título y autor del volumen: El regreso del muerto, Suleiman Cassamo. Traducción, introducción y notas de Joaquín García-Medall. Lengua de partida: portugués de Mozambique. Prólogo de Juan Zarandona, pp. 13-15. ISBN: 84-8448-091-7.

2001 Disbabelia. Colección Hermèneus de traducciones Ignotas. $\mathrm{N}^{\circ} 3$ (Valladolid: Servicio de Publicaciones e Intercambio Editorial - Universidad de Valladolid). Título y autor del volumen: Canciones populares neogriegas, de Nikolaos Politis. Traducción, introducción y notas de Román Bermejo LópezMuñiz. Lengua de partida: griego moderno. Prólogo de Amor López Jimeno, pp. 13-17. ISBN: 84-8448-095-X.

2002 Disbabelia. Colección Hermèneus de traducciones ignotas. $\mathrm{N}^{\circ} 4$ (Valladolid: Servicio de Publicaciones e Intercambio Editorial - Universidad de Valladolid). Título y autor del volumen: Cuentos populares búlgaros, anónimo. Traducción, introducción y notas de Denitza Bogomilova Atanassova. Lengua de partida: búlgaro. Prólogo de Ani Levi, pp. 13-15. ISBN: 84-8448-157-3.

2002 Disbabelia. Colección Hermèneus de traducciones ignotas. $\mathrm{N}^{\circ} 5$ (Valladolid: Servicio de Publicaciones e Intercambio Editorial - Universidad de Valladolid). Título y autor del volumen: Escritos desconocidos, de Ambrose G. Bierce. Traducción, introducción y notas de Sonia Santos Vila. Lengua de partida: inglés. Prólogo de Isabel Paraíso, pp. 15-16. ISBN: 84-8448-158-1.

2002 Disbabelia. Colección Hermèneus de traducciones ignotas. $\mathrm{N}^{\circ} 6$ (Valladolid: Servicio de Publicaciones e Intercambio Editorial - Universidad de Valladolid). Título y autor del volumen: Verano, de C.M. van den Heever. Traducción, introducción y notas de Santiago Martín y Juan Zarandona. Lengua de partida: afrikáans. Prólogo de Elize Botha, pp. 13-20. ISBN: 848448-185-9.

2003 Disbabelia. Colección Hermèneus de traducciones ignotas. $\mathrm{N}^{\circ} 7$ (Valladolid: Servicio de Publicaciones e Intercambio Editorial - Universidad de Valladolid). Título y autor del volumen: La leyenda de los tres Reyes Magos y 
ENTRECULTURAS Número 4. ISSN: 1989-5097. Fecha de publicación: 29-01-2012

Gregorio el de la Roca, de Johannes de Hildesheim y anónimo. Introducción, traducción y notas de María Teresa Sánchez. Lengua de partida: alemán. Prólogo de Feliciano Pérez Vargas, pp. 13-14. ISBN: 84-8448-209-X.

2004 Disbabelia. Colección Hermèneus de traducciones ignotas. $\mathrm{N}^{\circ} 8$ (Valladolid: Servicio de Publicaciones e Intercambio Editorial - Universidad de Valladolid). Título y autor del volumen: Es más fácil poner una pica en Flandes, de Barbara Noack. Introducción, traducción y notas de Carmen Gierden y Dirk Hofmann. Lengua de partida: alemán. Prólogo de Rafael López-Campos Bodienau, pp. 13-14. ISBN: 84-8448-271-5.

2004 Disbabelia. Colección Hermèneus de Traducciones Ignotas. $\mathrm{N}^{\circ} 9$ (Valladolid: Servicio de Publicaciones e Intercambio Editorial - Universidad de Valladolid). Títulos y autores del volumen: El conde de Gabalís, del Abate Montfaucon de Villars y El silfo, de Crébillon. Introducción, traducción y notas de María Teresa Ramos Gómez. Lengua de partida: francés. Prólogo de Francisco Hernández. ISBN: 84-8448-293-6.

2004 Disbabelia. Colección Hermèneus de Traducciones Ignotas. $\mathrm{N}^{\mathrm{o}} 10$ (Valladolid: Servicio de Publicaciones e Intercambio Editorial - Universidad de Valladolid). Título y autor del volumen: Erec, de Hartmann von Aue. Introducción de Marta Montero Navarro. Traducción y notas de Eva Parra Membrives. Lengua de partida: alemán. Prólogo de Juan Miguel Zarandona, pp. 13-15. ISBN: 84-8448-304-5.

2007 Disbabelia. Colección Hermèneus de Traducciones Ignotas. N $\mathrm{N}^{\circ} 11$ (Valladolid: Servicio de Publicaciones e Intercambio Editorial - Universidad de Valladolid). Título y autor del volumen: Libro del Rey Arturo. Según la parte artúrica del Roman de Brut de Wace. Introducción, traducción y notas de Mario Botero García. Lengua de partida: francés antiguo. Prólogo de Juan Miguel Zarandona, pp. 15-16. ISBN: 978-84-8448-404-2.

2007 Disbabelia. Colección Hermèneus de Traducciones Ignotas. $\mathrm{N}^{\mathrm{o}} 12$ (Valladolid: Servicio de Publicaciones e Intercambio Editorial - Universidad de Valladolid). Título y autor del volumen: Lírica medieval alemana con voz femenina (siglos XII-XIII), Anónimo. Introducción, traducción y notas de María Paz Muñoz-Saavedra y Juan Carlos Búa Carballo. Lengua de partida: alemán medieval. Prólogo de Victor Millet Schröder, pp. 19-21. ISBN: 978-84-8448398-4. 
2007 Disbabelia. Colección Hermèneus de Traducciones Ignotas. $\mathrm{N}^{\circ} 13$ (Valladolid: Servicio de Publicaciones e Intercambio Editorial - Universidad de Valladolid). Título y autor del volumen: Los Adioses de Arras, Jean Bodel, Baude Fastoul y Adam de la Halle. Introducción, traducción y notas de Antonia Martínez Pérez. Lengua de partida: francés medieval. Prólogo de Carlos Alvar, pp. 13-15. ISBN: 978-84-8448-405-9.

2007 Disbabelia. Colección Hermèneus de Traducciones Ignotas. $\mathrm{N}^{\circ} 14$ (Valladolid: Servicio de Publicaciones e Intercambio Editorial - Universidad de Valladolid). Título y autor del volumen: Sonetos de Crimea. Farys, Adam Mickiewicz. Traducción, introducción y notas de Antonio Benítez Burraco. Lengua de partida: polaco. Prólogo de Larisa V. Sokolova. ISBN: 978-84-8448418-9.

2009 Disbabelia. Colección Hermèneus de Traducciones Ignotas. $\mathrm{N}^{\mathrm{o}} 15$ (Valladolid: Servicio de Publicaciones e Intercambio Editorial - Universidad de Valladolid). Título y autor del volumen: Oswald de Múnich, Anónimo. Traducción, introducción y notas de Eva Parra Membrives y Miguel Ayerbe Linares. Lengua de partida: alemán. Prólogo de Albrecht Classen. ISBN: 97884-8448-501-8.

2010 Disbabelia. Colección Hermèneus de Traducciones Ignotas. $\mathrm{N}^{\circ} 16$ (Valladolid: Servicio de Publicaciones e Intercambio Editorial - Universidad de Valladolid). Título y autor del volumen: Historia de Campo Florido (Blómstrvalla saga), Anónimo. Traducción, introducción y notas de Mariano González Campo. Lengua de partida: noruego. Prólogo de Else Mundal. ISBN: 978-848448-549-0.

\section{CONCLUSIÓN}

Desde el Comité de Redacción de Hermēneus, creemos poder afirmar que los resultados: trece volúmenes publicados tanto de Hermèneus como de Vertere y dieciséis de Disbabelia (sin contar los dos en prensa que saldrán a la luz en 2011), son, en primer lugar, buena prueba de su existencia e interés por permanecer en el tiempo; e implican, en segundo término, una gran regularidad de tres publicaciones o colecciones muy consolidadas. También muestran que tenemos el compromiso de seguir adelante por el rentable camino emprendido en la búsqueda de nuevos logros que afiancen aún más lo ya logrado. Y todo ello en beneficio de los investigadores, estudiosos y traductores nacionales e internacionales que se decanten por nuestras colecciones. El Proyecto 
Hermēneus cuenta con su propia página web, bilingüe inglés-español, alojada en la página digital de la Universidad de Valladolid (www.uva.es/hermeneus) donde se publica todo lo que merece ser divulgado sobre el Proyecto Hermēneus. Permanezcan atentos.

Finalmente, también creemos que el Proyecto Hermēneus ha supuesto, en estos años, un modelo de mecenazgo cultural y científico de la Diputación de Soria y del Servicio de Publicaciones de la Universidad de Valladolid, así como de las personas que han conducido estas instituciones desde diferentes niveles de responsabilidad. Conviene recordarlo. 